Overlapping unit cells in 3D quasicrystal structure

This article has been downloaded from IOPscience. Please scroll down to see the full text article.

2006 J. Phys. A: Math. Gen. 399035

(http://iopscience.iop.org/0305-4470/39/29/001)

The Table of Contents and more related content is available

Download details:

IP Address: 150.203.32.127

The article was downloaded on 26/03/2010 at 00:31

Please note that terms and conditions apply. 


\title{
Overlapping unit cells in 3D quasicrystal structure
}

\author{
Helen Au-Yang and Jacques H H Perk \\ Department of Physics, Oklahoma State University, 145 Physical Sciences, Stillwater, \\ OK 74078-3072, USA \\ E-mail: perk@okstate.edu
}

Received 18 November 2005, in final form 5 March 2006

Published 5 July 2006

Online at stacks.iop.org/JPhysA/39/9035

\begin{abstract}
A three-dimensional quasiperiodic lattice, with overlapping unit cells and periodic in one direction, is constructed using grid and projection methods pioneered by de Bruijn. Each unit cell consists of 26 points, of which 22 are the vertices of a convex polytope $\mathcal{P}$, and 4 are interior points also shared with other neighbouring unit cells. Using Kronecker's theorem the frequencies of all possible types of overlapping are found.
\end{abstract}

PACS numbers: $05.50 .+\mathrm{q}, 61.44 . \mathrm{Br}$

(Some figures in this article are in colour only in the electronic version)

\section{Introduction}

Since the startling discovery of five-fold symmetry in quasiperiodic materials in 1984 [1], a great deal of research has been done on this subject by both physicists and mathematicians. Originally, quasicrystals were constructed by filling the space aperiodically with nonoverlapping tiles, such as in Penrose tilings [2-4]. ${ }^{1}$ However, recently, Gummelt [6] motivated by physical considerations proposed a description of quasicrystals in terms of overlappings of decagons. Further research [7-14] has shown that this may be a more sensible way to understand quasicrystalline materials - made of overlapping unit cells sharing atoms of nearby neighbours [8]. Here we use a multigrid method to produce a new example of three-dimensional overlapping unit cells.

\section{Multigrid construction}

It is well known that a Penrose tiling can be obtained by the projection of a slab of the 5D Euclidean lattice onto a particular $2 \mathrm{D}$ plane $\mathcal{D}[4,15,16]$, and hence its diffraction pattern [17-19] has ten-fold symmetry. It is also known that not all lattice points $k$ in $\mathbb{Z}^{5}$ are allowed

1 For a recent review of the theory of quasicrystals, see, e.g., [5]. 
(in the sense that they can be mapped onto vertices of a Penrose tiling); only those points whose projections into the three-dimensional orthogonal space $\mathcal{W}$ are inside the window of acceptance [15, 20] contribute. The window has been shown [15] to be the projection of the $5 \mathrm{D}$ unit cell $\mathrm{Cu}(5)$ with $2^{5}$ vertices into this $3 \mathrm{D}$ space $\mathcal{W}$. Each facet shared by two neighbouring 5D unit cell cubes is four dimensional and when projected into 3D space it produces a polyhedron $\mathcal{K}$ with 12 faces. Therefore, the projections of two adjacent 5D unit cells into 3D must share a common projected facet $\mathcal{K}$. Thus the idea of overlapping decagons must have its extension to three dimension, by projecting the 5D lattice into the space $\mathcal{W}$.

If $\boldsymbol{d}_{j}$ are the generators of the plane $\mathcal{D}$ and $\boldsymbol{w}_{j}$ are the generators of its orthogonal space $\mathcal{W}$, then the projection operators are the matrices $D^{\mathrm{T}}=\left(\boldsymbol{d}_{0}, \ldots, \boldsymbol{d}_{4}\right)$ and $\boldsymbol{W}^{\mathrm{T}}=\left(\boldsymbol{w}_{0}, \ldots, \boldsymbol{w}_{4}\right)$, such that $\boldsymbol{D}^{\mathrm{T}} \boldsymbol{W}=\boldsymbol{W}^{\mathrm{T}} \boldsymbol{D}=0$, where the superscript $\mathrm{T}$ denotes matrix transpose. More specifically, we choose

$$
\boldsymbol{d}_{j}^{\mathrm{T}}=(\cos j \theta, \sin j \theta), \quad \boldsymbol{w}_{j}^{\mathrm{T}}=(\cos 2 j \theta, \sin 2 j \theta, 1)=\left(\boldsymbol{d}_{2 j}^{\mathrm{T}}, 1\right),
$$

where $j=0, \ldots, 4$ and $\theta=2 \pi / 5$. Using notations and ideas introduced by de Bruijn [4], we consider the five grids consisting of bundles of equidistant planes defined by

$$
x \cos 2 j \theta+y \sin 2 j \theta+z+\gamma_{j}=\boldsymbol{w}_{j}^{\mathrm{T}} \boldsymbol{R}+\gamma_{j}=k_{j},
$$

for $j=0, \ldots, 4, k_{j} \in \mathbb{Z}$. In $(2), \boldsymbol{R}^{\mathrm{T}}=(x, y, z)$, and $\gamma_{j}$ are real numbers which shift the grids from the origin. We denote their sum by

$$
\gamma_{0}+\gamma_{1}+\gamma_{2}+\gamma_{3}+\gamma_{4}=c \text {. }
$$

Without loss of generality, we may restrict $c$ to $0 \leqslant c<1$.

It has been shown by de Bruijn [4] that the Penrose tiling associated with a 2D pentagrid has simple matching rules only for $c=0$. For $0<c<1$ the corresponding generalized Penrose tilings do not satisfy simple matching rules, and have seven different sets of vertices corresponding to the different intervals of $c[21,22]$. Nevertheless, the diffraction patterns are believed to be the same for all values of $c$ [23-25].

Let the integer $k_{j}$ be assigned to all points sandwiched between the grid planes defined by $k_{j}-1$ and $k_{j}$. Then, five integers

$$
K_{j}(\boldsymbol{R})=\left\lceil\boldsymbol{w}_{j}^{\mathrm{T}} \boldsymbol{R}+\gamma_{j}\right\rceil, \quad j=0, \ldots, 4,
$$

with $\lceil x\rceil$ the smallest integer greater than or equal to $x$, are uniquely assigned to every point $\boldsymbol{R}$ in $\mathbb{R}^{3}$. A mesh in $\mathbb{R}^{3}$ is now an interior volume, enclosed by grid planes, containing points with the same five integers. One next maps each mesh to a vertex in $\mathcal{W}$ by

$\boldsymbol{g}(\boldsymbol{R})=\sum_{j=0}^{4} K_{j}(\boldsymbol{R}) \boldsymbol{w}_{j}=\boldsymbol{W}^{\mathrm{T}} \boldsymbol{K}(\boldsymbol{R}), \quad \boldsymbol{K}^{\mathrm{T}}(\boldsymbol{R})=\left(K_{0}(\boldsymbol{R}), \ldots, K_{4}(\boldsymbol{R})\right)$.

The resulting collection of vertices $\mathcal{L}=\left\{\boldsymbol{g}(\boldsymbol{R}) \mid \boldsymbol{R} \in \mathbb{R}^{3}\right\}$ is a three-dimensional aperiodic lattice. It has been proven by de Bruijn [15] that a point $\boldsymbol{k}$ in $\mathbb{Z}^{5}$ satisfies the so-called mesh condition and therefore can be mapped into $\mathcal{L}$ if and only if $\boldsymbol{D}^{\mathrm{T}}(\boldsymbol{k}-\gamma)=\boldsymbol{D}^{\mathrm{T}} \boldsymbol{\lambda}$, where $\gamma^{\mathrm{T}}=\left(\gamma_{0}, \ldots, \gamma_{4}\right)$ and $\lambda^{\mathrm{T}}=\left(\lambda_{0}, \ldots, \lambda_{4}\right)$ with $0<\lambda_{j}<1$ so that $\boldsymbol{\lambda}$ is a point inside the 5D unit cube $\mathrm{Cu}(5)$. Thus, the window of acceptance is the interior of the convex hull of the points $\boldsymbol{D}^{\mathrm{T}} \boldsymbol{n}_{i}$, where $\boldsymbol{n}_{i}$ are the $2^{5}$ vertices of the 5D unit cube $\mathrm{Cu}(5)$, see figure 1 . We choose the $32 \boldsymbol{n}_{i}$ 's as follows:

$$
\begin{array}{ll}
\boldsymbol{n}_{0}=0, \quad \boldsymbol{n}_{31}=e_{0}+e_{1}+e_{2}+e_{3}+e_{4}, & \boldsymbol{n}_{j+1}=e_{1-2 j}, \\
\boldsymbol{n}_{j+6}=e_{1-2 j}+e_{4-2 j}, & \boldsymbol{n}_{j+11}=e_{1-2 j}+e_{2-2 j}, \\
\boldsymbol{n}_{j+16}=e_{1-2 j}+e_{2-2 j}+e_{5-2 j}, & \boldsymbol{n}_{j+21}=e_{1-2 j}+e_{2-2 j}+e_{4-2 j}, \\
\boldsymbol{n}_{j+26}=e_{1-2 j}+e_{2-2 j}+e_{4-2 j}+e_{5-2 j}, & (j=0, \ldots, 4),
\end{array}
$$




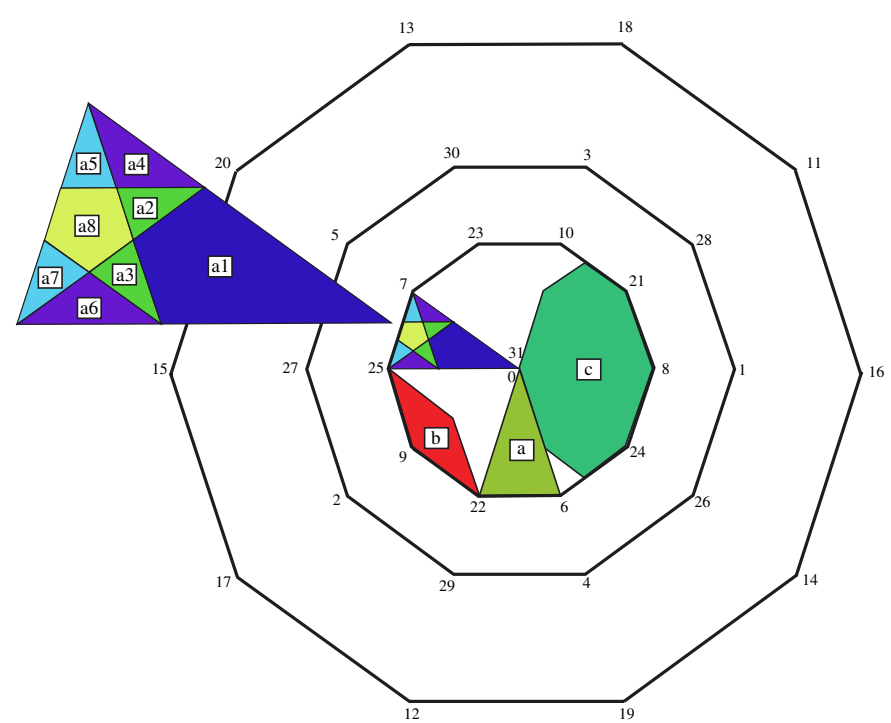

Figure 1. The projection of the $5 \mathrm{D}$ unit cube $\mathrm{Cu}(5)$ into the orthogonal $2 \mathrm{D}$ space $\mathcal{D}$. The window of acceptance is the interior of outer decagon $\mathcal{Q}$ whose vertices are given by (9). The innermost decagon is denoted by $\hat{\mathcal{Q}}$ and the middle decagon by $\overline{\mathcal{Q}}$ with vertices given by (10). There are ten triangles of type (a), which are all further subdivided into eight regions of type (a1), ., ( a8), as is indicated for one case in the magnification on the left. This is determined by possible overlaps of this triangle with the ten regions each of types (b) and (c), see the text.

where $e_{0}, \ldots, e_{4}$ are the standard unit vectors in $\mathbb{R}^{5}$, with subscripts counted mod 5 $\left(e_{j} \equiv e_{j \pm 5}\right)$.

The projection of $\mathrm{Cu}(5)$ with these 32 points into $\mathcal{W}$ is a polytope $\mathcal{P}$ with 40 edges connecting the 22 vertices, and with 20 faces. We let $\boldsymbol{P}_{i}=\boldsymbol{W}^{\mathrm{T}} \boldsymbol{n}_{i}$ for $i=0, \ldots, 31$. The bottom is $\boldsymbol{P}_{0}=(0,0,0)$ and top is $\boldsymbol{P}_{31}=(0,0,5)$; they are called the tips of the polytope. The remaining 20 vertices of $\mathcal{P}$ are

$$
\begin{array}{ll}
\boldsymbol{P}_{j+1}=\left(\boldsymbol{d}_{j}, 1\right), & \boldsymbol{P}_{j+6}=\left(\boldsymbol{d}_{j}+\boldsymbol{d}_{j+1}, 2\right), \\
\boldsymbol{P}_{j+21}=\left(-\boldsymbol{d}_{j-2}-\boldsymbol{d}_{j-1}, 3\right), & \boldsymbol{P}_{j+26}=\left(-\boldsymbol{d}_{j-1}, 4\right),
\end{array}
$$

for $j=0, \ldots, 4$. The other ten points $\boldsymbol{P}_{11}, \ldots, \boldsymbol{P}_{20}$ are in the interior of the polytope and are given by

$$
\boldsymbol{P}_{11+j}=\left(\boldsymbol{d}_{j}+\boldsymbol{d}_{j+2}, 2\right), \quad \boldsymbol{P}_{16+j}=\left(-\boldsymbol{d}_{j+1}-\boldsymbol{d}_{j-1}, 3\right),
$$

again for $j=0, \ldots, 4$.

The orthogonal projection of $\mathrm{Cu}(5)$ into $\mathcal{D}$ is a decagon $\mathcal{Q}$ with ten edges connecting the ten vertices, see figure 1 . Let $\boldsymbol{Q}_{i}=\boldsymbol{D}^{\mathrm{T}} \boldsymbol{n}_{i}$ for $i=0, \ldots, 31$. Then the vertices of the decagon are

$$
Q_{11+j}=-p d_{3-2 j}, \quad Q_{16+j}=p d_{5-2 j},
$$

with $j=0, \ldots, 4$, and $p=(\sqrt{5}+1) / 2$. The remaining 22 points $\boldsymbol{Q}_{0}, \ldots, \boldsymbol{Q}_{10}$ and $Q_{21}, \ldots, Q_{31}$ are in the interior; they are given by

$$
\begin{array}{ll}
\boldsymbol{Q}_{0}=\boldsymbol{Q}_{31}=0, & \boldsymbol{Q}_{j+1}=\boldsymbol{d}_{5-2 j}, \quad \boldsymbol{Q}_{26+j}=-\boldsymbol{d}_{2-2 j}, \\
\boldsymbol{Q}_{j+6}=p^{-1} \boldsymbol{d}_{4-2 j}, & \boldsymbol{Q}_{21+j}=-p^{-1} \boldsymbol{d}_{3-2 j} .
\end{array}
$$

Thus if the orthogonal projection $D^{\mathrm{T}}(\boldsymbol{k}-\gamma)$ is in $\mathcal{Q}$, then its projection $\boldsymbol{W}^{\mathrm{T}} \boldsymbol{k}$ is in $\mathcal{L}$. 
Consider a polytope $\mathcal{P}$ in $\mathcal{L}$ whose bottom is the projection of a point $\hat{\boldsymbol{k}}$ which is orthogonally projected onto the centre of the decagon, then the ten vertices of $\mathcal{P}$ at height $z=1$ or $z=4$ correspond to the vertices of the middle decagon $\overline{\mathcal{Q}}$, see figure 1 ; the ten vertices of the polytope at height $z=2$ or $z=3$ correspond to the vertices of the innermost decagon $\hat{\mathcal{Q}}$, while the ten interior points of the polytope correspond to the vertices of the (outer) decagon $\mathcal{Q}$ shown in figure 1 .

The points in the quasiperiodic lattice $\mathcal{L}$ need a careful analysis. We shall show that $\mathcal{L}$ consists of polytopes $\mathcal{P}$ with 22 vertices and 4 interior points which are shared with neighbouring polytopes. We shall also show that it is periodic in the $z$-direction-the direction of the line joining the two tips of $\mathcal{P}$-with period 5 corresponding to the height of $\mathcal{P}$.

\section{Overlapping unit cells}

The window $\mathcal{Q}$ is known $[4,26]$ to be everywhere dense and uniformly distributed. Thus each point in $\mathcal{Q}$ corresponds to a point in the quasiperiodic lattice $\mathcal{L}$. Using an idea of de Bruijn [4], we may find out the condition for both $\boldsymbol{k}$ and $\boldsymbol{k}+\boldsymbol{n}_{i}$ to satisfy the mesh condition-to lie both in the window of acceptance, which give insights to how points in $\mathcal{L}$ are related to one another.

It is straightforward to show that every point inside the innermost decagon $\hat{\mathcal{Q}}$ corresponds to a point in $\mathcal{L}$ that is connected with ten neighbours, and is in fact a tip of a polytope, as the middle decagon $\overline{\mathcal{Q}}$ whose centre is shifted to a point inside $\hat{\mathcal{Q}}$ still lies inside $\mathcal{Q}$. This innermost decagon $\hat{\mathcal{Q}}$ is further divided into ten triangles ${ }^{2}$. Whenever the centre of a decagon $\mathcal{Q}$ is shifted to a point inside one of the triangles, four of the vertices of the shifted outer decagon now lie inside $\mathcal{Q}$. More precisely, if $\boldsymbol{D}^{\mathrm{T}}(\boldsymbol{k}-\boldsymbol{\gamma})$ is inside triangle (a) in figure 1, then $\boldsymbol{W}^{\mathrm{T}} \boldsymbol{k}$ is a tip of a polytope $\mathcal{P}$ whose interior points $\boldsymbol{W}^{\mathrm{T}}\left(\boldsymbol{k}+\boldsymbol{n}_{20}\right), \boldsymbol{W}^{\mathrm{T}}\left(\boldsymbol{k}+\boldsymbol{n}_{13}\right)$, $\boldsymbol{W}^{\mathrm{T}}\left(\boldsymbol{k}+\boldsymbol{n}_{18}\right)$ and $\boldsymbol{W}^{\mathrm{T}}\left(\boldsymbol{k}+\boldsymbol{n}_{11}\right)$-corresponding to the four points on $\mathcal{Q}$ on the opposite side of triangle (a) - are now also in $\mathcal{L}$. This means each polytope in $\mathcal{L}$ can have only four interior points which are also in $\mathcal{L}$. Thus each such unit cell contains 26 atoms, 22 exterior and 4 interior sites.

It is also easy to find out how the polytopes share these interior points. This is equivalent to finding the condition that both $k$ and $k+n_{i}$ for $i=1, \ldots, 10$ or $i=21, \ldots, 30$ are orthogonally projected into $\hat{\mathcal{Q}}$.

Consider the ten vertices of the polytope at height $z=1$ or $z=4$ corresponding to the vertices of middle decagon $\overline{\mathcal{Q}}$. When the centre of the decagon is shifted to a point in one of the ten rhombs of type (b) shown in figure 1, then one of the ten vertices of the shifted $\overline{\mathcal{Q}}$ is inside $\hat{\mathcal{Q}}$. For example in figure $1, \boldsymbol{W}^{\mathrm{T}}(\boldsymbol{k})$ and $\boldsymbol{W}^{\mathrm{T}}\left(\boldsymbol{k}+\boldsymbol{n}_{28}\right)$ are both tips of polytopes, with $\boldsymbol{n}_{28}$ being the point on $\overline{\mathcal{Q}}$ on the opposite side of rhomb (b). As a consequence, the polytope whose top is $\boldsymbol{W}^{\mathrm{T}}\left(\boldsymbol{k}+\boldsymbol{n}_{28}\right)$ shares with the polytope $\mathcal{P}$ whose bottom is $\boldsymbol{W}^{\mathrm{T}}(\boldsymbol{k})$ a polyhedron $\mathcal{K}$ with 12 faces.

Moreover, the vertices of the polytope at $z=2$ or $z=3$ correspond to the vertices of $\hat{\mathcal{Q}}$. When the centre of this decagon is shifted to a point in one of the ten octagons of type (c) shown also in figure 1 , then one of the ten vertices of the shifted $\hat{\mathcal{Q}}$ moves inside $\hat{\mathcal{Q}}$. For example in figure $1, \boldsymbol{W}^{\mathrm{T}}(\boldsymbol{k})$ and $\boldsymbol{W}^{\mathrm{T}}\left(\boldsymbol{k}+\boldsymbol{n}_{25}\right)$ are then both tips of polytopes, while $\boldsymbol{n}_{25}$ is the point on $\hat{\mathcal{Q}}$ on the side opposite to the octagon. Such two polytopes share a polyhedron

2 A rotation of points in $\hat{\mathcal{Q}}$ by an angle of $2 \pi \ell / 5$ corresponds to a rotation of the polytopes in $\mathcal{L}$ by an angle of $4 \pi \ell / 5$ about the $z$-axis, while a rotation by $\pi$-inversion of points in $\hat{\mathcal{Q}}$ through the origin of decagon $\hat{\mathcal{Q}}$ —corresponds to the $3 \mathrm{D}$ inversion of polytopes in $\mathcal{L}$ through the centre of each polytope. 
$\mathcal{J}$ with six faces. When vertices of $\mathcal{P}$ at both $z=3$ and $z=4$ are inside $\hat{\mathcal{Q}}$, they are tops of some polytopes ${ }^{3}$ that share with $\mathcal{P}$ either a polyhedron $\mathcal{K}$ or $\mathcal{J}$.

By considering how these 20 regions intersect, we find that each of the triangles in $\hat{\mathcal{Q}}$ is further divided into eight regions, as shown for one case in figure 1, with this triangle magnified on the left. When the orthogonal projection of $\boldsymbol{k}-\boldsymbol{\gamma}$ is in (a1), its projection into $\mathcal{W}$ is a polytope $\mathcal{P}$ intersecting with four others whose tips are at $\boldsymbol{W}^{\mathrm{T}}\left(\boldsymbol{k}+\boldsymbol{n}_{21}\right), \boldsymbol{W}^{\mathrm{T}}\left(\boldsymbol{k}+\boldsymbol{n}_{8}\right)$, $\boldsymbol{W}^{\mathrm{T}}\left(\boldsymbol{k}+\boldsymbol{n}_{24}\right)$ and $\boldsymbol{W}^{\mathrm{T}}\left(\boldsymbol{k}+\boldsymbol{n}_{6}\right)$, and sharing with each of them a polyhedron of type $\mathcal{J}$. When the projection is in (a2) or (a3), either $\boldsymbol{W}^{\mathrm{T}}\left(\boldsymbol{k}+\boldsymbol{n}_{26}\right)$ or $\boldsymbol{W}^{\mathrm{T}}\left(\boldsymbol{k}+\boldsymbol{n}_{1}\right)$ becomes also a tip of a polytope, so that $\mathcal{P}$ intersects with five polytopes, sharing with one of them a polyhedron of type $\mathcal{K}$. In (a8), $\mathcal{P}$ intersects with all of the above six polytopes. In regions (a5) or (a7), $\boldsymbol{W}^{\mathrm{T}}\left(\boldsymbol{k}+\boldsymbol{n}_{21}\right)$ or $\boldsymbol{W}^{\mathrm{T}}\left(\boldsymbol{k}+\boldsymbol{n}_{6}\right)$ is no longer a tip and $\mathcal{P}$ intersects with five polytopes sharing with two of them a polyhedron of type $\mathcal{K}$. Finally, while in (a4) or (a6), either $\boldsymbol{W}^{\mathrm{T}}\left(\boldsymbol{k}+\boldsymbol{n}_{1}\right)$ or $\boldsymbol{W}^{\mathrm{T}}\left(\boldsymbol{k}+\boldsymbol{n}_{26}\right)$ is no longer a tip, and $\mathcal{P}$ intersects with four polytopes sharing with one of them a polyhedron $\mathcal{K}$ and with the other three a $\mathcal{J}$.

Therefore, in summary, there are only five different possibilities:

(i) The points inside a quadrilateral of type (a1) correspond to a polytope intersecting with four other polytopes sharing with each a polyhedron of type $\mathcal{J}$. An example of this case is shown in figure $2(a)$.

(ii) Points inside triangles of type (a2) or (a3) correspond to a polytope intersecting with five other polytopes, sharing with one of them a polyhedron $\mathcal{K}$ and with the other four polyhedra of type $\mathcal{J}$. Such a case is shown in figure $2(b)$.

(iii) If the point is inside triangles of type (a4) or (a6), the polytope intersects with four other polytopes sharing with one of them a polyhedron $\mathcal{K}$ and with the other three polyhedra of type $\mathcal{J}$.

(iv) If the point is in a triangle (a5) or (a7), the polytope intersects with five other polytopes sharing with two of them polyhedra of type $\mathcal{K}$ and with the other three polyhedra $\mathcal{J}$.

(v) Finally, if the point is inside a pentagon (a8), the polytope intersects with six other polytopes sharing with two of them a $\mathcal{K}$ and with the other four a $\mathcal{J}$, as is shown in figure 3 .

The relative frequencies are related to the ratios of their areas and therefore the normalized probabilities are given by

$$
\begin{aligned}
& P_{\mathrm{a} 1}=2 p^{-3}, \quad P_{\mathrm{a} 2}=P_{\mathrm{a} 3}=p^{-6}, \quad P_{\mathrm{a} 4}=P_{\mathrm{a} 6}=p^{-5}, \\
& P_{\mathrm{a} 5}=P_{\mathrm{a} 7}=p^{-6}, \quad P_{\mathrm{a} 8}=p^{-5}+p^{-7} .
\end{aligned}
$$

\section{Parallelepipeds and windows}

Now the condition that $\boldsymbol{k}$ satisfies the mesh condition can be further simplified by considering parallelepiped $P\left(k_{4}, k_{0}, k_{1}\right)$ sandwiched between the six grid planes $k_{4}-1, k_{4}, k_{0}-1, k_{0}, k_{1}-1$ and $k_{1}$. Using (2) for the grid planes, we can solve for the points of intersections, and find that for every point $\boldsymbol{R}$ in $P\left(k_{4}, k_{0}, k_{1}\right)$, we may write

$$
\begin{aligned}
& K_{0}(\boldsymbol{R})=k_{0}, \quad K_{1}(\boldsymbol{R})=k_{1}, \quad K_{2}(\boldsymbol{R})=\lfloor\alpha\rfloor+k_{4}+m, \\
& K_{3}(\boldsymbol{R})=\lfloor\beta\rfloor+k_{1}+n, \quad K_{4}(\boldsymbol{R})=k_{4},
\end{aligned}
$$

\footnotetext{
3 We shall later show that lattice $\mathcal{L}$ is periodic in the $z$-direction with period 5 .
} 


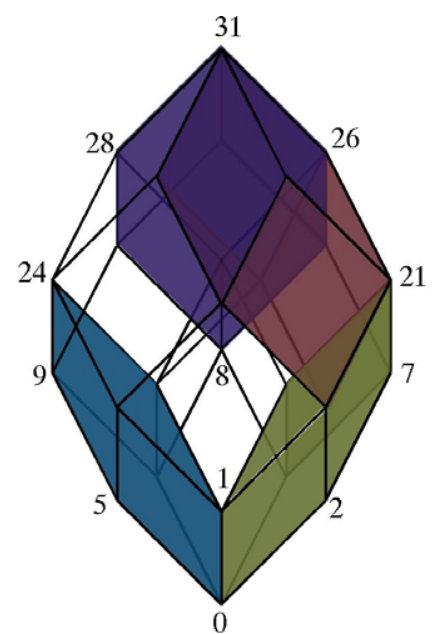

(a)

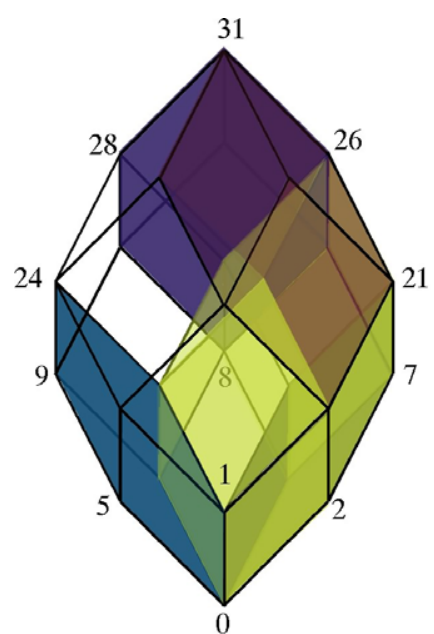

(b)

Figure 2. (a) When the orthogonal projection of $\boldsymbol{k}-\boldsymbol{\gamma}$ is in region (a1), its projection in $\mathcal{L}$ is a polytope sharing an interior point with each of the four neighbouring polytopes whose tips are projections of $\boldsymbol{k}+\boldsymbol{n}_{21}, \boldsymbol{k}+\boldsymbol{n}_{8}, \boldsymbol{k}+\boldsymbol{n}_{24}$ and $\boldsymbol{k}+\boldsymbol{n}_{6}$; the shared interior points are projections of $\boldsymbol{k}+n_{11}, k+n_{16}, k+n_{14}$ and $k+n_{19}$. (b) When it is in region (a2), the polytope has five neighbouring polytopes sharing the same interior points. The additional polytope has its top at $\boldsymbol{W}^{\mathrm{T}}\left(\boldsymbol{k}+\boldsymbol{n}_{26}\right)$.

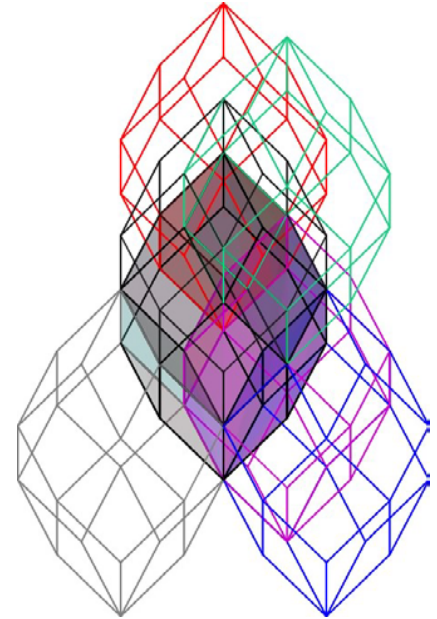

(a)

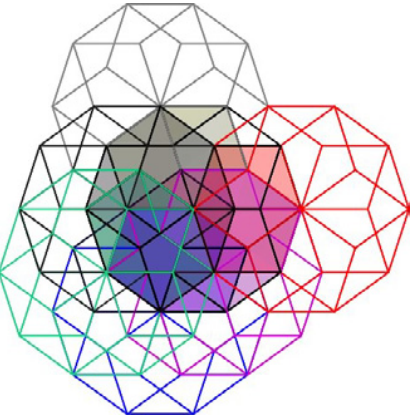

$(b)$

Figure 3. The polytope $\mathcal{P}$ and its six neighbours in $\mathcal{L}$ for case (a8). Their intersections with $\mathcal{P}$ are indicated with different colours. In (a) the orientation is $(\theta, \phi)=\left(0,80^{\circ}\right)$; in (b) $(\theta, \phi)=\left(90^{\circ}, 0\right)$, so that each polytope appears as a decagon.

where $\lfloor x\rfloor$ is the greatest integer less than or equal to $x$, while $m$ and $n$ are integers satisfying $-1 \leqslant m, n \leqslant 2$ and

$$
\begin{gathered}
\alpha=p^{-1}\left(k_{0}-k_{1}-\gamma_{0}+\gamma_{1}\right)+\gamma_{2}-\gamma_{4}, \\
\beta=p^{-1}\left(k_{0}-k_{4}-\gamma_{0}+\gamma_{4}\right)+\gamma_{3}-\gamma_{1} .
\end{gathered}
$$


The range of $m$ and $n$ in equation (12) is limited by their possible values at the eight corners of the parallelepiped, but not all 16 choices are allowed by the mesh condition. Therefore, we now project $\boldsymbol{K}(\boldsymbol{R})$ given by (12) to $\mathcal{D}$ and find

$$
\boldsymbol{D}^{\mathrm{T}}(\boldsymbol{K}(\boldsymbol{R})-\gamma)=\sum_{j=0}^{4}\left(K_{j}(\boldsymbol{R})-\gamma_{j}\right) \boldsymbol{d}_{j}=(m-a) \boldsymbol{d}_{2}+(n-b) \boldsymbol{d}_{3},
$$

in which $a \equiv\{\alpha\} \equiv \alpha-\lfloor\alpha\rfloor$ and $b \equiv\{\beta\} \equiv \beta-\lfloor\beta\rfloor$. The vector (14) must lie within the decagon $\mathcal{Q}$. Thus the allowed values of $m$ and $n$ are determined by $a$ and $b$ only. As the differences $k_{0}-k_{4}$ and $k_{0}-k_{1}$ run through all integer values, we find from Kronecker's theorem [27] that $a$ and $b$ are everywhere dense and uniformly distributed in the interval $(0,1)$. Proofs of such 'ergodicity' in more general situations can be found, e.g., in the works of Hof and Schlottmann [28, 29].

\section{Periodicity in third direction}

Furthermore, because of the difference property shown in (13), $\alpha$ and $\beta$, which determine the configuration of the parallelepiped, remain the same, if $\left(k_{0}, k_{1}, k_{4}\right) \rightarrow\left(k_{0}+\ell, k_{1}+\ell, k_{4}+\ell\right)$. As a consequence we find $\boldsymbol{K}(\boldsymbol{R}) \rightarrow \boldsymbol{K}(\boldsymbol{R})+\ell \boldsymbol{n}_{31}$, and its projection is periodic in the $z$-direction with period equal to 5. It is also interesting to note that if $\gamma \rightarrow \gamma-\frac{1}{5} c n_{31}, \alpha$ and $\beta$ in (13) are also unchanged, so that the projection into the 3D space does not show drastic changes when $c \neq 0$, which is behaviour very different from the 2D case [21].

It is not difficult to find values of $(a-m, b-n)$ in (14) corresponding to the ten vertices of the decagon $\mathcal{Q}$ given by (9). We find

$$
\begin{array}{lll}
Q_{16} \leftrightarrow(a-0, b-0)=(1,1), & Q_{11} \leftrightarrow(a-0, b+1)=(0, p), \\
Q_{18} \leftrightarrow(a-1, b+1)=(-1, p), & Q_{13} \leftrightarrow(a-2, b+1)=(-p, 1), \\
Q_{20} \leftrightarrow(a-2, b-0)=(-p, 0), & Q_{15} \leftrightarrow(a-1, b-1)=(0,-1), \\
Q_{17} \leftrightarrow(a-1, b-2)=(0,-p), & Q_{12} \leftrightarrow(a-0, b-2)=(1,-p), \\
Q_{19} \leftrightarrow(a+1, b-2)=(p,-1), & Q_{14} \leftrightarrow(a+1, b-1)=(p, 0),
\end{array}
$$

up to ambiguities when $a$ or $b$ is integer, as $m$ or $n$ changes by 1 when choosing $a$ or $b$ to be 0 or 1 . The edges of decagon $\mathcal{Q}$ lead to linear equations in $(a-m, b-n)$ and the mesh condition for $\boldsymbol{K}(\boldsymbol{R})$ becomes a set of inequalities in $a-m$ and $b-n$ as shown in figure 4 . Hence, it is very easy to create a routine to generate $\mathcal{L}$.

We note that $\mathcal{L}$ is periodic in the longitudinal or $z$-direction with period 5, and aperiodic in the horizontal directions. This is a model with five layers which repeat in the $z$-direction periodically. In each of the layers, the points behave similarly, and the allowed sites for $z=3$ are shown in figure 5(a). This shows decagonal symmetry, but the layer may not be covered by Penrose tiles. In figure $5(b)$, we have sites for atoms in all five layers plotted, with sites in different layers represented by different symbols and colours.

Therefore, $\mathcal{L}$ is another example of a decagonal quasicrystal. Decagonal quasicrystals exist in nature, and have been extensively studied experimentally and theoretically [30-35]. From Steurer's review [31], one finds translational periods along the ten-fold $z$-axis varying from $4 \AA$ to $16 \AA$ for different alloys, which may allow two layers [33], or even five or more layers, within a period.

A situation somewhat similar to ours is found by Ben-Abraham, Lerer and Snapir [14], who find that the projection of a $6 \mathrm{D}$ lattice to $3 \mathrm{D}$ for a certain choice of bases produces a 


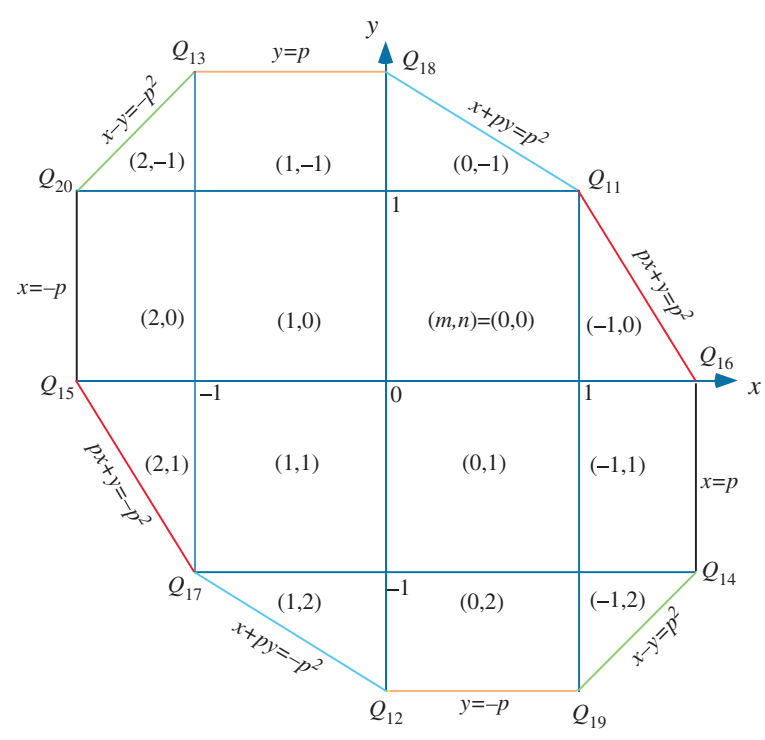

Figure 4. The image of $\mathcal{Q}$ under the inverse mapping of (14) into the $(a-m, b-n)$ plane. The boundaries of regions with different $(m, n)$ are given by linear equations in terms of $x \equiv a-m$ and $y \equiv b-n$.

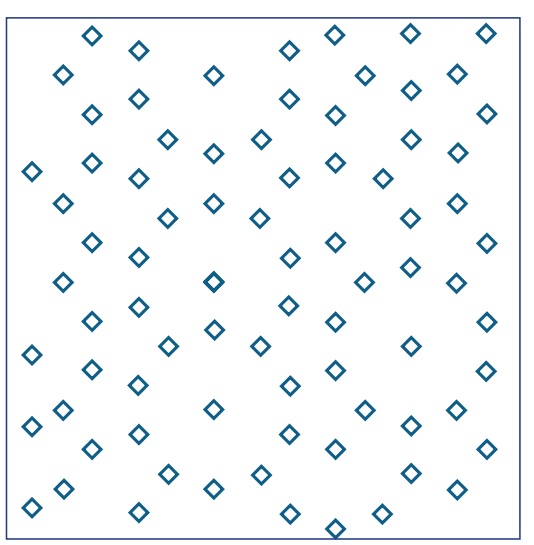

(a)

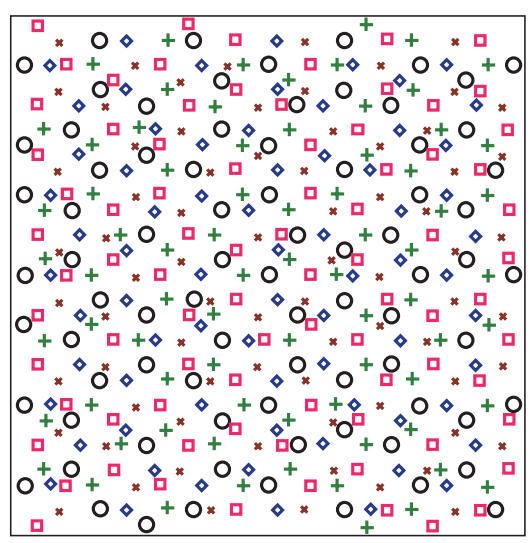

(b)

Figure 5. The allowed positions in each layer. (a) Allowed sites at height $z=3$ are plotted. (b) All layers are plotted: points for $z=1$ are represented by black boxes; for $z=2$ by red circles; for $z=3$ by blue diamonds; for $z=4$ by green crosses; for $z=5$ by brown points.

quasicrystal lattice which is also periodic in the $z$-direction. Their model has six-fold rather than five-fold symmetry.

The projection of $\mathbb{Z}^{5} \rightarrow \mathbb{R}^{3}$ is well known [30, 34, 36]. However, interpreting the $3 \mathrm{D}$ quasicrystal lattice $\mathcal{L}$ as overlapping polytopes $\mathcal{P}$ provides a more systematic way to understand this lattice. We find that each unit cell has 26 sites, sharing the four interior sites with its neighbours. The lattice is periodic in the $z$-direction with period 5 , and quasiperiodic in the $x y$-directions. Unlike the projection of $\mathbb{Z}^{5} \rightarrow \mathbb{R}^{2}$, which undergoes drastic change in 
behaviour (no inflation and deflation rules) if $c \neq 0$, the projections into 3D are in the same class for $c=0$ and $c \neq 0$.

Since real quasicrystals have icosahedra or triacontahedra as unit cells, which are projections of a six-dimensional hypercube to a 3D space [10], the above method perhaps can also be used to determine all possible overlappings and their frequencies.

\section{Acknowledgments}

We are most grateful to Dr M Widom, Dr C Richard and Dr M Baake for providing us with many useful references. Comments on our manuscript by Dr M Widom and Dr M Baake are also much appreciated.

\section{References}

[1] Shechtman D, Blech I, Gratias D R and Cahn J W 1984 Metallic phase with long-range orientational order and no translational symmetry Phys. Rev. Lett. 53 1951-3

[2] Penrose R 1989 Tilings and quasi-crystals; a non-local growth problem? Introduction to The Mathematics of Quasicrystals (Aperiodicity and Order vol 2) ed M V Jarić (Boston: Academic) pp 53-79

[3] Grünbaum B and Shephard G C 1987 Tilings and Patterns (New York: W H Freeman and Co) chapter 10

[4] de Bruijn N G 1981 Algebraic theory of Penrose's non-periodic tilings of the plane. I Indagationes Math. 84 39-52

de Bruijn N G 1981 Algebraic theory of Penrose's non-periodic tilings of the plane. II Indagationes Math. 84 53-66

[5] Kramer P and Papadopolos Z (eds) 2002 Coverings of Discrete Quasiperiodic Sets (Berlin: Springer)

[6] Gummelt P 1996 Penrose tilings as coverings of congruent decagons Geometriae Dedicata 62 1-17

[7] Steinhardt P J and Jeong H C 1996 A simpler approach to Penrose tilings with implications for quasicrystal formation Nature 382 433-5

[8] Steinhardt P J, Jeong H C, Saitoheong K, Tanaka M, Abe E and Tsai A P 1996 Experimental verification of the quasi-unit-cell model of quasicrystal structure Nature 396 55-7

[9] Lord E A and Ranganathan S 2001 The Gummelt decagon as a 'quasi unit cell' Acta Crystallogr. A 57 531-9

[10] Lord E A, Ranganathan S and Kulkarni U D 2001 Quasicrystals: tiling versus clustering Phil. Mag. A $812645-51$

[11] Kramer P 2002 Covering of discrete quasiperiodic sets: concepts and theory Coverings of Discrete Quasiperiodic Sets ed P Kramer and Z Papadopolos (Berlin: Springer) pp 1-21

[12] Duneau M and Gratias D 2002 Covering clusters in icosahedral quasicrystals Coverings of Discrete Quasiperiodic Sets ed P Kramer and Z Papadopolos (Berlin: Springer) pp 23-61

[13] Gähler F, Gummelt P and Ben-Abraham S I 2002 Generation of quasiperiodic order by maximal cluster covering Coverings of Discrete Quasiperiodic Sets ed P Kramer and Z Papadopolos (Berlin: Springer) pp 63-95

[14] Ben-Abraham S I, Lerer Y and Snapir Y 2004 Constructing a dodecagonal structure by projection in two stages J. Non-Cryst. Solids 334\&335 71-6

[15] de Bruijn N G 1986 Quasicrystal and their Fourier transform Indagationes Math. 89 123-52

[16] Gähler F and Rhyner J 1986 Equivalence of the generalised grid and projection methods for the construction of quasiperiodic tilings J. Phys. A: Math. Gen. $19267-77$

[17] Elser V 1986 The diffraction pattern of projected structures Acta Crystallogr. A 42 36-43

[18] Duneau M and Katz A 1985 Quasiperiodic patterns Phys. Rev. Lett. 54 2688-91

[19] Mackay A L 1982 Crystallography on the Penrose pattern Physica A 114 609-13

[20] Baake M, Joseph D, Kramer P and Schlottmann M 1990 Root lattices and quasicrystals J. Phys. A: Math. Gen. 23 L1037-41

[21] Au-Yang H and Perk J H H 2005 Generalized Penrose tilings in preparation

[22] Au-Yang H and Perk J H H 2005 Quasicrystals: projection of 5d lattice into 2 or 3 dimensions Preprint math-ph/0606028

[23] Levine D and Steinhardt P J 1986 Quasicrystals: I. Definition and structure Phys. Rev. B 34 596-616

[24] Socolar J E S and Steinhardt P J 1986 Quasicrystals: II. Unit-cell configurations Phys. Rev. B 34 617-47

[25] Ishihara K N and Yamamoto A 1988 Penrose patterns and related structures: I. Superstructure and generalized Penrose patterns Acta Crystallogr. A 44 508-16 
[26] de Bruijn N G 1992 Penrose patterns are almost entirely determined by two points Discrete Math. 106/107 97-104

[27] Hardy G H and Wright E M 1960 An Introduction to the Theory of Numbers 4th edn (London: Oxford University Press) chapter XXIII Kronecker's Theorem

[28] Hof A 1998 Uniform distribution and the projection method Quasicrystals and Discrete Geometry ed J Patera (Providence RI: American Mathematical Society) pp 201-6

[29] Schlottmann M 1998 Cut-and-project sets in locally compact abelian groups Quasicrystals and Discrete Geometry ed J Patera (Providence RI: American Mathematical Society) pp 247-64

[30] Yamamoto A and Ishihara K N 1988 Penrose patterns and related structures: II. Decagonal quasicrystals Acta Crystallogr. A 44 707-14

[31] Steurer W 1990 The structure of quasicrystals Z. Kristallogr. 190 179-234

[32] Steurer W and Kuo K H 1990 Five-dimensional structure analysis of decagonal $\mathrm{Al}_{65} \mathrm{Cu}_{20} \mathrm{Co}_{15}$ Acta Crystallogr. B 46 703-12

[33] Cockayne E and Widom M 1998 Ternary model of an Al-Cu-Co decagonal quasicrystal Phys. Rev. Lett. 81 598-601

[34] Steurer W and Haibach T 1999 The periodic average structure of particular quasicrystals Acta Crystallogr. A $5548-57$

[35] Cervellino A, Haibach T and Steurer W 2002 Structure solution of the basic decagonal Al-Co-Ni phase by the atomic surfaces modelling method Acta Crystallogr. B 58 8-33

[36] Kramer P 2002 Voronoi and Delone clusters in dual quasiperiodic tilings Coverings of Discrete Quasiperiodic Sets ed P Kramer and Z Papadopolos (Berlin: Springer) pp 185-225 